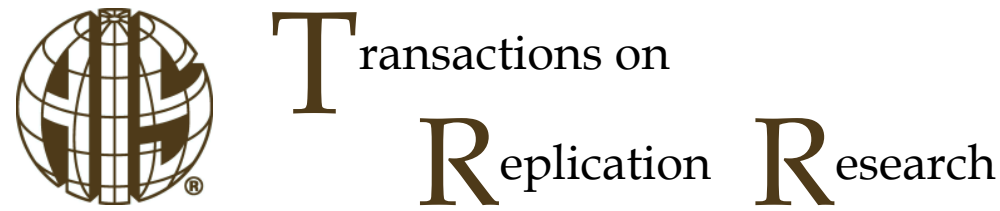

\section{Replication Research: Campus Emergency Notification Systems}

Rishikesh Chetan Muchhala

Management Consultant - PricewaterhouseCoopers LLP, USA

muchhalarishikesh@gmail.com

\author{
Patricia L. Moravec \\ McCombs School of Business \\ The University of Texas at Austin \\ patricia.moravec@mccombs.utexas.edu
}

\begin{abstract}
:
This paper is an exact replication of the Han, Ada, Sharman and Rao (2015) article on Campus Emergency Notification Systems (ENS). In their study, Information Quality Trust and Subjective Norm were the factors which most commonly induced recipients of ENS notifications to comply with the information and instructions in the notification; Perceived Safety Threat, Perceived Financial Threat, and Past Experience also played a role for some types of ENS notifications. We found essentially the same results, although there were some differences. In our study, Information Quality Trust was again the most important determinant. Subjective norms played a role, but were not a fundamentally important factor as they were in the Han, et al. study; we speculate this may be due to different cultures between our participants and theirs. The other three factors were important for some types of ENS notifications but not others. Our research also suggests that future research should consider past experience with ENS notifications, such as the frequency, location relevance, and the timeliness of past ENS notifications received.
\end{abstract}

Keywords: Compliance, campus alerts, emergency notification systems, information quality trust, study replication

The manuscript was originally received on 07/11/2016 and was with the authors for 36 months for 3 revisions. 


\section{Introduction}

In recent years, there have been a number of emergency incidents such as crimes, extreme weather conditions, and accidents, on university campuses all across United States (Mittroff, Diamond and Alpaslam, 2006). The United States Federal Bureau of Investigation (FBI) reports crime and security statistics on US university campuses as required under the Clery Act. Data from 2013-2015 indicate that on average across all of the universities in the US there are about 2000 fires, 14500 burglaries/robberies/thefts, 20 murders, 2300 aggravated assaults, and 5000 sexual assaults each year ${ }^{1}$. In response to these events, many universities have adopted Emergency Notification Systems (ENS) to either warn or inform students, faculty and staff affiliated with the university about such incidents (Han, Ada, Sharman, and Rao, 2015). Yet, merely having such systems in place does not persuade the recipients to believe in and comply with the information and instructions provided by the system.

A recent study by Han, et al. (2015) showed that while most students said they would comply with most ENS notifications, about $40 \%$ said they would verify the information in the ENS notification before acting. In certain time-sensitive situations, if the user fails to comply immediately, precious time is wasted (Han, et al., 2015) and the risk may be similar to non-compliance (e.g., waiting until you see the shooter in an active shooter situation before seeking shelter). If a substantial minority of recipients choose to not comply immediately, it defeats the purpose of employing ENS. Hence, it is important to understand what factors influence the decision to comply with ENS notifications.

Han, et al. (2015) examined three fundamentally different theoretical routes that organizations can use to influence organizational members to comply with its requests (Etzioni, 1961; Parsons, 1957). The first is coercive power, such as sanctions. Few universities will choose to impose sanctions for non-compliance, but one way to use coercive power is to emphasize threats to physical safety in the ENS notification (Han, et al. 2015). The second route is remunerative power, such as rewards. Once again, few universities will choose to offer rewards for compliance, but one way to use remunerative power is to emphasize threats to finances in the ENS notification (Han, et al. 2015). The third route is norms; universities can exert pressure to change the subjective norms around the compliance decision (Han, et al. 2015).

The Han, et al. (2015) study provides some initial understanding of the factors that influence whether students will pay attention to ENS notifications. They found that subjective norms and the extent to which students trusted the information quality of the ENS dominated the decision to comply or not. Safety and financial concerns played a role in only a few specific circumstances. Thus the take-aways from this initial study are for universities to focus on providing good quality, timely information via the ENS and to change organizational culture, so that subjective norms are to immediately comply with ENS notifications. But, these conclusions are based on only one study of students living in one specific region of the US. To what extent are these conclusions replicable? Should we ignore safety and financial issues as sources of influence is all but a few specific situations?

\subsection{The Original Study}

The Han, et al., (2015) study used a "survey-collecting service company" to distribute an online survey to university students in "the northern region of the United States" (p. 917), and received 821 responses. They presented each participant with one of five scenarios that described an incident (snowstorm, active shooter, building-related (fire), health-related (epidemic), and robbery) and an ENS notification with a call to action. Participants were asked whether they would comply immediately, verify the information in the ENS notification before complying, or ignore the ENS notification. Less than $1 \%$ of participants reported they would ignore the ENS notification, so those responses were omitted from the analysis.

The study examined the effects of five factors that influenced the decision to comply with the ENS notification. Table 1 presents the results of their study. In general, information quality trust and subjective norm increased compliance in most situations. A perceived safety threat was important for fast developing situations (i.e., active shooter and building fire) but not for slow developing incidents such as snowstorms, health, or robbery (students reported that ENS notifications for robberies were often sent long after the

${ }^{1}$ see https://ope.ed.gov/campussafety/\#/datafile/list 
incident, so they were not perceived to be fast developing incidents). Perceived financial safety was important for a building fire situation, perhaps because a fire has the potential to harm property (but not for a robbery because ENS notifications for robberies were perceived to be after the fact). Past experience with an incident was also a factor, likely because students have more experience with fires from fire drills than other situations (Han, et al., 2015).

\begin{tabular}{|c|c|c|c|c|c|c|}
\hline & Test Statistics & $\begin{array}{l}\text { Regression Model 1: } \\
\text { DV - Snowstorm } \\
\text { Incident }\end{array}$ & $\begin{array}{l}\text { Regression Model 2: DV } \\
\text { - Active Shooter Incident }\end{array}$ & $\begin{array}{l}\text { Regression Model 3: DV } \\
\text { - Building-Related } \\
\text { Incident }\end{array}$ & $\begin{array}{l}\text { Regression Model 4: DV } \\
\text { - Health-Related } \\
\text { Incident }\end{array}$ & $\begin{array}{l}\text { Regression Model 5: DV - } \\
\text { Robbery Incident }\end{array}$ \\
\hline $\begin{array}{c}\text { Past } \\
\text { Experience }\end{array}$ & $\begin{array}{c}\beta \\
\mathrm{SE}_{\beta} \\
\text { Wald's } \chi^{2} \\
\text { Odd's Ratio } \\
95 \% \mathrm{CI} \\
\end{array}$ & $\begin{array}{c}0.10 \\
0.38 \\
0.06 \\
1.10 \\
0.52-2.32 \\
\end{array}$ & $\begin{array}{c}-0.32 \\
0.42 \\
0.58 \\
0.73 \\
0.32-1.64 \\
\end{array}$ & $\begin{array}{c}1.02 \\
0.48 \\
4.64 \\
2.58^{*} \\
1.02-6.47 \\
\end{array}$ & $\begin{array}{c}-0.32 \\
0.35 \\
0.81 \\
0.73 \\
0.36-1.46 \\
\end{array}$ & $\begin{array}{c}0.38 \\
0.57 \\
0.45 \\
1.47 \\
0.48-4.50 \\
\end{array}$ \\
\hline $\begin{array}{l}\text { Subjective } \\
\text { Norm }\end{array}$ & $\begin{array}{c}\beta \\
\mathrm{SE}_{\beta} \\
\text { Wald's } \chi^{2} \\
\text { Odd's Ratio } \\
95 \% \mathrm{CI} \\
\end{array}$ & $\begin{array}{c}0.01 \\
0.01 \\
3.87 \\
1.01 * \\
1.01-1.02 \\
\end{array}$ & $\begin{array}{c}0.01 \\
0.01 \\
7.60 \\
1.01 * * \\
1.00-1.02 \\
\end{array}$ & $\begin{array}{c}-0.01 \\
0.01 \\
0.92 \\
0.99 \\
0.98-1.01\end{array}$ & $\begin{array}{c}0.01 \\
0.01 \\
4.19 \\
1.01 * \\
1.01-1.02 \\
\end{array}$ & $\begin{array}{c}0.02 \\
0.01 \\
6.29 \\
1.02 * \\
1.00-1.03 \\
\end{array}$ \\
\hline $\begin{array}{l}\text { Perceived } \\
\text { Safety } \\
\text { Threat }\end{array}$ & $\begin{array}{c}\beta \\
\mathrm{SE}_{\beta} \\
\text { Wald's } \chi^{2} \\
\text { Odd's Ratio } \\
95 \% \mathrm{CI} \\
\end{array}$ & $\begin{array}{c}0.42 \\
0.28 \\
2.32 \\
1.53 \\
0.89-2.64 \\
\end{array}$ & $\begin{array}{c}0.60 \\
0.29 \\
4.22 \\
1.83^{*} \\
1.03-3.25 \\
\end{array}$ & $\begin{array}{c}0.63 \\
0.31 \\
4.06 \\
1.87^{*} \\
1.02-3.45 \\
\end{array}$ & $\begin{array}{c}-0.14 \\
0.28 \\
0.24 \\
0.87 \\
0.50-1.51 \\
\end{array}$ & $\begin{array}{c}-0.90 \\
0.50 \\
3.21 \\
0.41 \\
0.15-1.09 \\
\end{array}$ \\
\hline $\begin{array}{l}\text { Perceived } \\
\text { Financial } \\
\text { Threat }\end{array}$ & $\begin{array}{c}\beta \\
\mathrm{SE}_{\beta} \\
\text { Wald's } \chi^{2} \\
\text { Odd's Ratio } \\
95 \% \mathrm{CI} \\
\end{array}$ & $\begin{array}{c}-0.35 \\
0.26 \\
1.72 \\
0.72 \\
0.43-1.18 \\
\end{array}$ & $\begin{array}{c}0.08 \\
0.22 \\
0.12 \\
1.08 \\
0.70-1.65 \\
\end{array}$ & $\begin{array}{c}0.54 \\
0.26 \\
4.32 \\
1.71 * \\
1.03-2.83 \\
\end{array}$ & $\begin{array}{c}0.22 \\
0.25 \\
0.76 \\
1.25 \\
0.76-2.05 \\
\end{array}$ & $\begin{array}{c}0.27 \\
0.35 \\
0.60 \\
1.32 \\
0.66-2.63 \\
\end{array}$ \\
\hline $\begin{array}{c}\text { Information } \\
\text { Quality } \\
\text { Trust }\end{array}$ & $\begin{array}{c}\beta \\
\mathrm{SE}_{\beta} \\
\text { Wald's } \chi^{2} \\
\text { Odd's Ratio } \\
95 \% \mathrm{CI} \\
\end{array}$ & $\begin{array}{c}0.60 \\
0.27 \\
4.80 \\
1.81 * \\
1.07-3.09 \\
\end{array}$ & $\begin{array}{c}0.66 \\
0.30 \\
4.87 \\
1.93^{*} \\
1.08-3.47 \\
\end{array}$ & $\begin{array}{c}0.76 \\
0.34 \\
4.93 \\
2.15^{*} \\
1.10-4.23 \\
\end{array}$ & $\begin{array}{c}1.13 \\
0.32 \\
12.31 \\
3.11^{* *} \\
1.65-5.86 \\
\end{array}$ & $\begin{array}{c}0.91 \\
0.43 \\
4.40 \\
2.49 * \\
1.06-5.82 \\
\end{array}$ \\
\hline \multicolumn{2}{|c|}{$\begin{array}{l}\text { Response Ratio (Comply } \\
\text { Immediately: Verify First) }\end{array}$} & $90: 74$ & 101:63 & $105: 59$ & $78: 86$ & $104: 61$ \\
\hline \multicolumn{2}{|c|}{$-2 \mathrm{LL}$} & 167.67 & 148.09 & 142.03 & 162.70 & 94.36 \\
\hline \multicolumn{2}{|r|}{$\chi^{2}$} & $26.94 * *$ & $40.03^{* *}$ & $36.68^{* *}$ & $37.76^{* *}$ & $24.00^{* *}$ \\
\hline \multicolumn{2}{|c|}{ Nagelkerke $\mathrm{R}^{2}$} & 0.23 & 0.32 & 0.32 & 0.31 & 0.28 \\
\hline \multicolumn{2}{|c|}{$\begin{array}{c}\text { Overall Correct } \\
\text { Classification }\end{array}$} & $68.1 \%$ & $78.3 \%$ & $78.8 \%$ & $66.9 \%$ & $90.0 \%$ \\
\hline
\end{tabular}

${ }^{*} p<0.05 ;{ }^{* *} p<0.01$ 


\section{Methodology}

In this study, we replicated the Han, et al. (2015) study almost exactly. We invited undergraduate students enrolled in a large section business course at a large US state university in the Midwest to participate in an online survey. We invited 699 students to participate in the survey and we received 538 total responses (a response rate of $77 \%$ ). $64 \%$ were male, with $38 \% 19$ years of age or less, $61 \% 20$ to 24 , and $1 \% 25$ or older. $80 \%$ were US citizens, with $68 \%$ being Caucasian, $25 \%$ Asian, $4 \%$ Hispanic, and $1 \%$ black, native American or other ethnicity. Students received extra credit for participating.

The survey was conducted online through the Qualtrics software. Each student was presented with all five incident descriptions in a random order to minimize order effects; the Han, et al., (2015) study was a between-subjects study in which each subject received only one incident description. The median time taken to complete the survey was 9.2 minutes. The five incident descriptions were taken directly from Han, et al. (2015) and adapted slightly for our context (e.g., by using names of buildings on our campus). The incident descriptions can be found in Table 2.

\begin{tabular}{|c|c|}
\hline \multicolumn{2}{|r|}{ Table 2. Incident Descriptions } \\
\hline Incident & Explanation \\
\hline $\begin{array}{l}\text { Incident 1: } \\
\text { Snowstorm }\end{array}$ & $\begin{array}{l}\text { Snowstorms are storms where large amounts of snow fall. Two inches }(5 \mathrm{~cm}) \text { of snow is enough } \\
\text { to create serious disruptions to traffic and school transport in many school districts across the } \\
\text { United States. There may be power loss in a snowstorm. Roads may be rendered impassable. } \\
\text { Students may be alerted to stay indoors and avoid driving. } \\
\text { Jeff and Tom are roommates who live a } 40 \text {-minute drive away from school. One morning, they } \\
\text { were going to university campus together to study for their final exam. Jeff was driving, and Tom } \\
\text { received a notification message from campus alert on his phone. It said, "A snowstorm is } \\
\text { approaching campus. A driving ban is in effect. Shelter in place and avoid driving until further } \\
\text { notice." }\end{array}$ \\
\hline $\begin{array}{l}\text { Incident } 2: \\
\text { Active shooter }\end{array}$ & $\begin{array}{l}\text { These incidents involve one or more shooters on campus or suspects with weapons on campus. } \\
\text { Examples of such incidents include the Virginia Tech campus shooting incident, which took place } \\
\text { on April 16, 2007. Thirty-two students were affected in that incident. During such incidents, } \\
\text { students may be alerted to find a safe place and lock the door or take other action. } \\
\text { One day, Jeff was studying in the } \\
\text { "A suspected gunman is on campus. Immediately go to the nearest room and lock the door. Keep } \\
\text { quiet until further notice." }\end{array}$ \\
\hline $\begin{array}{l}\text { Incident } \\
\text { Building- } \\
\text { related incident }\end{array}$ & $\begin{array}{l}\text { These incidents include fires and gas leaks and typically require evacuation of the premises or } \\
\text { buildings. Building fires are quite common on college campuses. Examples: One student died } \\
\text { due to a fire incident at the University of California-Los Angeles on December 29, 2009; two } \\
\text { students were injured in a fire incident on January, 30, 2010, at Western State College of Colorado. } \\
\text { Students may be required to evacuate the building immediately. } \\
\text { One day, Jeff was going to meet his classmate to discuss a project at 3:00 p.m. At 2:55 p.m., } \\
\text { while he was waiting for the classmate on the second floor in the Library, he received a } \\
\text { campus emergency notification: "Smoke was observed on the third floor in the Library. All } \\
\text { personnel should leave the building immediately using stairs." }\end{array}$ \\
\hline $\begin{array}{l}\text { Incident 4: } \\
\text { Health-related } \\
\text { incident }\end{array}$ & $\begin{array}{l}\text { These incidents include outbreaks such as the swine flu epidemic and water contamination. } \\
\text { Students may be required to contact health care providers if they notice any symptoms. } \\
\text { Jeff was working on campus one day when he received a message from the campus alert system: } \\
\text { "Disease outbreak reported in carty. If you are coughing, sneezing, or having a fever, see } \\
\text { a health care provider immediately." Jeff had been coughing for a couple of days. }\end{array}$ \\
\hline $\begin{array}{l}\text { Incident 5: } \\
\text { Robbery } \\
\text { incident }\end{array}$ & $\begin{array}{l}\text { These incidents involve the taking of or attempt to take anything of value from the care, custody, } \\
\text { or control of a person or persons by force or threat of force or violence and/or by putting the victim } \\
\text { in a state of fear. Example: A student was robbed at gunpoint on the main campus of the } \\
\text { University at Buffalo. Students may be advised to avoid walking alone in the area. } \\
\text { Jeff was preparing to walk home from the Library to his dorm when he received a message: } \\
\text { "A robbery incident was reported at } 9 \text { p.m. near Library. Please be cautious and avoid } \\
\text { poorly lit areas." }\end{array}$ \\
\hline
\end{tabular}

Note: Specific building and city names were redacted for review purposes

The survey used exactly the same three-part item to measure compliance with the ENS notification (comply immediately, verify first before complying, or ignore) as well as the same items to create the five factors 
(information quality trust, subjective norm, perceived safety threat, perceive financial threat, and past experience). The items are presented in the Appendix A. The Cronbach's alpha for all the indicated adequate reliability $(>0.7)$; see Table 3 .

\begin{tabular}{|l|l|l|l|l|l|l|l|}
\hline \multicolumn{7}{|c|}{ Table 3. Cronbach's Alpha } \\
\hline $\begin{array}{l}\text { Constructs } \\
\text { For Cronbach's alpha }\end{array}$ & $\begin{array}{l}\text { Incident 1 } \\
\text { Snowstorm }\end{array}$ & $: \begin{array}{l}\text { Incident 2 } \\
\text { Active } \\
\text { Shooter }\end{array}$ & $\begin{array}{l}\text { Incident 3 } \\
\text { Building } \\
\text { Related }\end{array} \quad \begin{array}{l}\text { Incident } 4 \\
\text { Health } \\
\text { Related }\end{array} \quad \begin{array}{l}\text { Incident } \\
\text { Robbery }\end{array}$ \\
\hline Motivation to Comply & 0.855 & 0.914 & 0.898 & 0.884 & 0.910 \\
Normative Belief & 0.843 & 0.947 & 0.922 & 0.894 & 0.905 \\
Perceived Safety Threat & 0.938 & 0.900 & 0.948 & 0.863 & 0.947 \\
Perceived Financial Threat & 0.949 & 0.968 & 0.970 & 0.939 & 0.930 \\
Information Reliability Trust & 0.868 & 0.870 & 0.858 & 0.883 & 0.879 \\
Information Action-ability Trust & 0.871 & 0.802 & 0.888 & 0.871 & 0.853 \\
Information Criticality Trust & 0.896 & 0.892 & 0.871 & 0.867 & 0.880 \\
\hline
\end{tabular}

The correlations among the factors are presented in Table 4. We note that some of the correlations are high, indicating the potential for multicollinearity. We present one test for this in the Post-Hoc Tests section of the Results.

\begin{tabular}{|c|c|c|c|c|c|}
\hline \multicolumn{6}{|c|}{ Table 4. Correlation Matrix } \\
\hline $\begin{array}{l}\text { Pearson } \\
\text { Correlation }\end{array}$ & Variable & $\begin{array}{l}\text { (1) } \\
\text { Subjective } \\
\text { Norm }\end{array}$ & $\begin{array}{l}\text { (2) Perceived } \\
\text { Safety Threat }\end{array}$ & $\begin{array}{l}\text { (3) Perceived } \\
\text { Financial } \\
\text { Threat }\end{array}$ & $\begin{array}{l}\text { (4) } \\
\text { Information } \\
\text { Quality Trust }\end{array}$ \\
\hline $\begin{array}{l}\text { Incident } 1: \\
\text { Snowstorm }\end{array}$ & $\begin{array}{l}\text { (1) Subjective Norm } \\
\text { (2) Perceived Safety Threat } \\
\text { (3) Perceived Financial Threat } \\
\text { (4) Information Quality Trust }\end{array}$ & $\begin{array}{l}1 \\
0.605 \\
0.298 \\
0.719 \\
\end{array}$ & $\begin{array}{l}1 \\
0.387 \\
0.700 \\
\end{array}$ & $\begin{array}{l}1 \\
0.372\end{array}$ & 1 \\
\hline $\begin{array}{l}\text { Incident 2: } \\
\text { Active Shooter }\end{array}$ & $\begin{array}{l}\text { (1) Subjective Norm } \\
\text { (2) Perceived Safety Threat } \\
\text { (3) Perceived Financial Threat } \\
\text { (4) Information Quality Trust }\end{array}$ & $\begin{array}{l}1 \\
0.605 \\
0.117 \\
0.626 \\
\end{array}$ & $\begin{array}{l}1 \\
0.076 \\
0.615 \\
\end{array}$ & $\begin{array}{l}1 \\
0.107 \\
\end{array}$ & 1 \\
\hline $\begin{array}{l}\text { Incident 3: } \\
\text { Building Related }\end{array}$ & $\begin{array}{l}\text { (1) Subjective Norm } \\
\text { (2) Perceived Safety Threat } \\
\text { (3) Perceived Financial Threat } \\
\text { (4) Information Quality Trust }\end{array}$ & $\begin{array}{l}1 \\
0.608 \\
0.176 \\
0.639 \\
\end{array}$ & $\begin{array}{l}1 \\
0.257 \\
0.625 \\
\end{array}$ & $\begin{array}{l}1 \\
0.195 \\
\end{array}$ & 1 \\
\hline $\begin{array}{l}\text { Incident } \\
\text { Health Related }\end{array}$ & $\begin{array}{l}\text { (1) Subjective Norm } \\
\text { (2) Perceived Safety Threat } \\
\text { (3) Perceived Financial Threat } \\
\text { (4) Information Quality Trust }\end{array}$ & $\begin{array}{l}1 \\
0.555 \\
0.329 \\
0.707 \\
\end{array}$ & $\begin{array}{l}1 \\
0.446 \\
0.639 \\
\end{array}$ & $\begin{array}{l}1 \\
0.414 \\
\end{array}$ & 1 \\
\hline $\begin{array}{l}\text { Incident } \quad 5: \\
\text { Robbery }\end{array}$ & $\begin{array}{l}\text { (1) Subjective Norm } \\
\text { (2) Perceived Safety Threat } \\
\text { (3) Perceived Financial Threat } \\
\text { (4) Information Quality Trust }\end{array}$ & $\begin{array}{l}1 \\
0.662 \\
0.341 \\
0.710\end{array}$ & $\begin{array}{l}1 \\
0.451 \\
0.720\end{array}$ & $\begin{array}{l}1 \\
0.423\end{array}$ & 1 \\
\hline
\end{tabular}




\section{Results}

The dependent variable has three actions choices: comply immediately, verify first, or ignore. In the original study, less than $1 \%$ of participants reported they would ignore the notification, so Han, et al., (2015) chose to omit those data and focus only on participants who reported they would comply immediately or verify first. In our study, a meaningful number of participants chose to ignore the notifications $(14.10 \%$ for snowstorm, $1.80 \%$ for active shooter, $3.90 \%$ for building-related, $15.50 \%$ for health-related, and $12.50 \%$ for Robbery). Therefore, we present our results in two separate analyses. The first is following the approach of Han, et al., (2015) and omitting those who chose to ignore the notification (a two-outcome model using only comply and verify). The second is including those who chose to ignore the notification (a three-outcome model including comply, verify, and ignore).

Table 5 presents the two outcome model results. Han, et al. used five separate logistic regressions (one for each incident), as did we. These analyses indicate that Information Quality Trust was the most important factor influencing intention to comply, as it was significant for four of the five incidents (at $p=.05$ ). Perceived Safety Threat was significant for three incidents (active shooter, building fire, robbery). Subjective Norm was significant for snowstorm and active shooter. Perceived Financial Threat was significant for the snowstorm and the health incident. Past Experience with an incident was not significant for any of the incidents.

\begin{tabular}{|c|c|c|c|c|c|c|}
\hline & Test Statistics & $\begin{array}{l}\text { S1: } \\
\text { Snowstorm }\end{array}$ & $\begin{array}{l}\text { S2: Active } \\
\text { Shooter }\end{array}$ & $\begin{array}{l}\text { S3: Building } \\
\text { Related }\end{array}$ & $\begin{array}{l}\text { S4: Health } \\
\text { Related }\end{array}$ & S5: Robbery \\
\hline \multirow{5}{*}{$\begin{array}{l}\text { Past } \\
\text { Experience }\end{array}$} & $\beta$ & -0.136 & 0.031 & -0.077 & -0.234 & 0.130 \\
\hline & $\mathrm{SE}_{\beta}$ & 0.380 & 0.466 & 0.393 & 0.409 & 0.408 \\
\hline & Wald's $X^{2}$ & 0.127 & 0.004 & 0.038 & 0.328 & 0.101 \\
\hline & Odds Ratio & 0.873 & 1.032 & 0.926 & 0.791 & 1.139 \\
\hline & $95 \% \mathrm{Cl}$ & $0.414-1.840$ & $0.414-2.571$ & $0.429-2.000$ & $0.355-1.764$ & $0.512-2.534$ \\
\hline \multirow{5}{*}{$\begin{array}{l}\text { Subjective } \\
\text { Norm }\end{array}$} & $\beta$ & -0.038 & -0.034 & -0.015 & 0.007 & -0.023 \\
\hline & $\mathrm{SE}_{\beta}$ & 0.015 & 0.014 & 0.013 & 0.015 & 0.015 \\
\hline & Wald's $X^{2}$ & 6.014 & 5.676 & 1.219 & 0.232 & 2.301 \\
\hline & Odds Ratio & 0.963 & 0.967 & 0.985 & 1.007 & 0.977 \\
\hline & $95 \% \mathrm{Cl}$ & $0.934-0.992$ & $0.940-0.994$ & $0.960-1.011$ & $0.978-1.038$ & $0.949-1.007$ \\
\hline \multirow{5}{*}{$\begin{array}{l}\text { Perceived } \\
\text { Safety Threat }\end{array}$} & $\beta$ & -0.092 & -0.316 & -0.652 & -0.081 & -0.357 \\
\hline & $\mathrm{SE}_{\beta}$ & 0.129 & 0.154 & 0.126 & 0.119 & 0.125 \\
\hline & Wald's $X^{2}$ & 0.508 & 4.240 & 26.922 & 0.466 & 8.104 \\
\hline & Odds Ratio & 0.912 & 0.729 & 0.521 & 0.922 & 0.700 \\
\hline & $95 \% \mathrm{Cl}$ & $0.708-1.175$ & $0.540-0.985$ & $0.407-0.666$ & $0.730-1.164$ & $0.547-0.895$ \\
\hline \multirow{5}{*}{$\begin{array}{l}\text { Perceived } \\
\text { Financial } \\
\text { Threat }\end{array}$} & $\beta$ & -0.172 & 0.009 & -0.003 & -0.319 & -0.053 \\
\hline & $\mathrm{SE}_{\beta}$ & 0.065 & 0.070 & 0.063 & .079 & 0.068 \\
\hline & Wald's $X^{2}$ & 6.988 & 0.018 & 0.003 & 16.518 & 0.608 \\
\hline & Odds Ratio & 0.842 & 1.010 & 0.997 & 0.727 & 0.949 \\
\hline & $95 \% \mathrm{Cl}$ & $0.741-0.956$ & $0.880-1.158$ & $0.881-1.128$ & $0.623-0.848$ & $0.831-1.083$ \\
\hline \multirow{5}{*}{$\begin{array}{l}\text { Information } \\
\text { Quality Trust }\end{array}$} & $\beta$ & -0.280 & -0.960 & -0.370 & -0.657 & -0.404 \\
\hline & $\mathrm{SE}_{\beta}$ & 0.178 & 0.181 & 0.149 & 0.185 & 0.163 \\
\hline & Wald's X ${ }^{2}$ & 2.491 & 28.029 & 6.170 & 12.565 & 6.175 \\
\hline & Odds Ratio & 0.756 & 0.383 & 0.691 & 0.519 & 0.668 \\
\hline & $95 \% \mathrm{Cl}$ & $0.538-1.070$ & $0.268-0.546$ & $0.519-0.925$ & $0.361-0.746$ & $0.485-0.918$ \\
\hline \multicolumn{2}{|c|}{$\begin{array}{l}\text { Response Ratio (Comply } \\
\text { Immediately : Verify First }\end{array}$} & $164: 273$ & $388: 112$ & $307: 184$ & $152: 280$ & $291: 155$ \\
\hline \multicolumn{2}{|c|}{$-2 \mathrm{LL}$} & 528.048 & 412.162 & 549.338 & 490.839 & 508.301 \\
\hline \multicolumn{2}{|l|}{$x^{2}$} & 50.285 & 119.762 & 100.189 & 69.539 & 67.848 \\
\hline \multicolumn{2}{|c|}{ Nagelkerke $\mathrm{R}^{2}$} & 0.148 & 0.325 & 0.252 & 0.205 & 0.195 \\
\hline \multicolumn{2}{|c|}{ Overall Correct Classification } & $68.60 \%$ & $77.60 \%$ & $72.90 \%$ & $72.50 \%$ & $72.20 \%$ \\
\hline
\end{tabular}

Table 6 presents the three outcome model results. Because we had three outcomes, we used five separate multinomial logistic regressions with a base case of ignore the ENS notifications. These analyses indicate that Information Quality Trust was the most important factor influencing intention to comply, as it was significant for all five incidents (at $p=.05$ ). Perceived Safety Threat was significant for three incidents (active shooter, building fire, robbery). Past Experience with an incident was significant for snowstorm and building 
fire. Subjective Norm was significant for snowstorm and robbery. Perceived Financial Threat was significant for the health incident.

\begin{tabular}{|c|c|c|c|c|c|c|c|c|c|c|c|}
\hline \multicolumn{12}{|c|}{$\begin{array}{l}\text { Table 6. Three-Outcome Results (Comply Immediately vs. Verify First vs. Ignore). } \\
\text { Gray cells are statistically significant at } p=.05\end{array}$} \\
\hline & \multirow[t]{2}{*}{ Test Statistics } & \multicolumn{2}{|c|}{ S1: Snowstorm } & \multicolumn{2}{|c|}{ S2: Active Shooter } & \multicolumn{2}{|c|}{ S3: Building Related } & \multicolumn{2}{|c|}{ S4: Health Related } & \multicolumn{2}{|c|}{ S5: Robbery } \\
\hline & & CIm & VFTC & $\mathrm{CIm}$ & VFTC & $\mathrm{CIm}$ & VFTC & CIm & VFTC & $\mathrm{CIm}$ & VFTC \\
\hline \multirow{5}{*}{$\begin{array}{c}\text { Past } \\
\text { Experience }\end{array}$} & $\beta$ & -1.878 & -1.819 & -0.956 & -0.964 & -14.827 & -14.748 & -0.182 & 0.096 & -0.438 & -0.558 \\
\hline & $\mathrm{SE}_{\beta}$ & 0.934 & 0.833 & 1.444 & 1.389 & 0.393 & 0.000 & 0.603 & 0.518 & 0.691 & 0.640 \\
\hline & Wald's $\chi^{2}$ & 4.044 & 4.247 & 0.438 & 0.482 & 1421.741 & - & 0.091 & 0.034 & 0.402 & 0.760 \\
\hline & Odd's Ratio & 0.153 & 0.162 & 0.384 & 0.381 & $3.64 \mathrm{E}-07$ & $3.94 \mathrm{E}-07$ & 0.834 & 1.100 & 0.645 & 0.572 \\
\hline & $95 \% \mathrm{CI}$ & $0.025-0.954$ & $0.029-0.915$ & $0.023-6.518$ & $0.025-5.801$ & $1.68 \mathrm{E}-7-7.86 \mathrm{E}-7$ & 3.93E-7-3.93E-7 & $0.256-2.716$ & $0.399-3.038$ & $0.167-2.498$ & $0.163-2.007$ \\
\hline \multirow{5}{*}{$\begin{array}{l}\text { Subjective } \\
\text { Norm }\end{array}$} & $\beta$ & 0.107 & 0.068 & -0.041 & -0.076 & -0.012 & -0.027 & -0.023 & -0.016 & 0.058 & 0.034 \\
\hline & $\mathrm{SE}_{\beta}$ & 0.024 & 0.021 & 0.056 & 0.055 & 0.034 & 0.033 & 0.023 & 0.020 & 0.022 & 0.021 \\
\hline & Wald's $\chi^{2}$ & 20.171 & 10.787 & 0.528 & 1.887 & 0.124 & 0.640 & 0.969 & 0.624 & 6.730 & 2.562 \\
\hline & Odd's Ratio & 1.113 & 1.070 & 0.960 & 0.927 & 0.988 & 0.974 & 0.978 & 0.955 & 1.060 & 1.034 \\
\hline & $95 \% \mathrm{CI}$ & $1.062-1.166$ & $1.028-1.114$ & 0.861-1.071 & $0.832-1.033$ & $0.924-1.057$ & $0.912-1.040$ & $0.935-1.023$ & $0.947-1.023$ & 1.014-1.107 & $0.992-1.078$ \\
\hline \multirow{5}{*}{$\begin{array}{c}\text { Perceived } \\
\text { Safety Threat }\end{array}$} & $\beta$ & 0.213 & 0.150 & 1.320 & 0.974 & 1.142 & 0.480 & 0.300 & 0.201 & 0.700 & 0.341 \\
\hline & $\mathrm{SE}_{\beta}$ & 0.172 & 0.142 & 0.426 & 0.416 & 0.244 & 0.220 & 0.164 & 0.134 & 0.179 & 0.162 \\
\hline & Wald's $\chi^{2}$ & 1.525 & 1.116 & 9.591 & 5.477 & 21.972 & 4.768 & 3.337 & 2.254 & 15.286 & 4.416 \\
\hline & Odd's Ratio & 1.237 & 1.162 & 3.744 & 2.648 & 3.132 & 1.616 & 1.350 & 1.223 & 2.013 & 1.407 \\
\hline & $95 \% \mathrm{CI}$ & $0.882-1.735$ & $0.879-1.537$ & $1.624-8.633$ & 1.171-5.984 & $1.943-5.049$ & $1.050-2.485$ & $0.978-1.864$ & $0.940-1.589$ & $1.418-2.859$ & $1.023-1.934$ \\
\hline \multirow{5}{*}{$\begin{array}{l}\text { Perceived } \\
\text { Financial } \\
\text { Threat }\end{array}$} & $\beta$ & 0.167 & -0.006 & 0.019 & 0.032 & -0.155 & -0.161 & 0.361 & 0.059 & -0.046 & -0.090 \\
\hline & $\mathrm{SE}_{\beta}$ & 0.111 & 0.101 & 0.321 & 0.320 & 0.177 & 0.173 & 0.126 & 0.111 & 0.123 & 0.121 \\
\hline & Wald's $\chi^{2}$ & 2.266 & 0.004 & 0.004 & 0.010 & 0.770 & 0.869 & 8.263 & 0.285 & 0.137 & 0.557 \\
\hline & Odd's Ratio & 1.182 & 0.994 & 1.019 & 1.033 & 0.856 & 0.851 & 1.435 & 1.061 & 0.955 & 0.914 \\
\hline & $95 \% \mathrm{CI}$ & $0.951-1.470$ & 0.816-1.210 & $0.544-1.911$ & $0.552-1.932$ & $0.606-1.271$ & $0.607-1.194$ & $1.122-1.836$ & $0.854-1.318$ & $0.750-1.217$ & 0.721-1.158 \\
\hline \multirow{5}{*}{$\begin{array}{c}\text { Information } \\
\text { Quality Trust }\end{array}$} & $\beta$ & 0.684 & 0.364 & 1.818 & 0.885 & 1.200 & 0.842 & 1.646 & 1.001 & 1.029 & 0.631 \\
\hline & $\mathrm{SE}_{\beta}$ & 0.244 & 0.198 & 0.414 & 0.389 & 0.275 & 0.259 & 0.259 & 0.208 & 0.232 & 0.213 \\
\hline & Wald's $\chi^{2}$ & 7.892 & 3.364 & 19.269 & 5.166 & 19.003 & 10.588 & 40.306 & 23.139 & 19.662 & 8.816 \\
\hline & Odd's Ratio & 1.982 & 1.439 & 6.161 & 2.423 & 3.321 & 2.320 & 5.185 & 2.722 & 2.798 & 1.880 \\
\hline & $95 \% \mathrm{CI}$ & $1.230-3.195$ & $0.975-2.122$ & $2.736-13.876$ & $1.130-5.196$ & $1.936-5.697$ & $1.398-3.852$ & $3.119-8.617$ & $1.810-4.093$ & $1.776-4.409$ & $1.239-2.852$ \\
\hline \multicolumn{2}{|c|}{ Response Ratio* } & \multicolumn{2}{|c|}{$164: 273: 72$} & \multicolumn{2}{|c|}{ 388:112:9 } & \multicolumn{2}{|c|}{$307: 184: 20$} & \multicolumn{2}{|c|}{$152: 280: 79$} & \multicolumn{2}{|c|}{$291: 155: 64$} \\
\hline \multicolumn{2}{|c|}{ Ignore percentage } & \multicolumn{2}{|c|}{$14.10 \%$} & \multicolumn{2}{|c|}{$1.80 \%$} & \multicolumn{2}{|c|}{$3.90 \%$} & \multicolumn{2}{|c|}{$15.50 \%$} & \multicolumn{2}{|c|}{$12.50 \%$} \\
\hline \multicolumn{2}{|c|}{$-2 \mathrm{LL}$} & \multicolumn{2}{|c|}{805.004} & 433. & & 610. & 090 & & 602 & & \\
\hline & $x^{2}$ & & & 160. & & 157. & 903 & & 455 & & \\
\hline Nagelk & zerke $\mathrm{R}^{2}$ & & & 0.3 & & 0.3 & & & 43 & & \\
\hline Overall Correc & ct classification & & & 80.0 & & 71.2 & $0 \%$ & & & & \\
\hline
\end{tabular}

*Response Ratio $=$ comply immediately $:$ verify first and then comply $:$ ignore

\subsection{Post-Hoc Tests}

We had some concerns about the potential for multicolinearity given the high correlations in Table 4, but logistic regression provides no statistics for this. However, standard linear regression provides the variance inflation factor (VIF). A VIF greater than 10 indicates multicollinearity may be an issue (Kutner, Nachtsheim, and Neter, 2004). Standard linear regression can be used to approximate logistic regression when the dependent variable has only two outcomes (Kutner, et al, 2004). As a post-hoc test of the potential impact of multicollinearity, we ran five standard linear regression analyses on our two-outcome data and found all VIFs to be below 2.5, indicating no multicollinearity issues.

As another post-hoc test, we re-ran all 10 models again ( 5 two-outcome and 5 three-outcome) but this time using only Information Quality Trust as the sole independent variable and found that the correct classification 
percentages decreased by an average of only 3.1 percentage points. In other words, the information trust only models were able to correctly predict students' compliance intentions within three percentage points; all other factors combined improved the classification accuracy by only three percentage points.

An inspection of the information quality trust data suggested that there might be some differences in trust for different scenarios, so we used a repeated measures general linear model to check for differences. We found that there were significant differences in information quality trust due to the scenario $(F(1,508)=$ $23,876.74, p<.000$ ). Table 7 presents the means and the groups that were significantly different (using LSD pairwise comparison). Students had the greatest trust in the information quality of the ENS for active shooter incidents and building related incidents (i.e., fire). They had less trust in ENS reports of robbery or snowstorm incidents and less again in reports of health-related incidents.

\begin{tabular}{|l|l|l|}
\hline \multicolumn{3}{|c|}{$\begin{array}{c}\text { Table 7. Significantly Different Groups of Mean } \\
\text { Information Quality Trust by Scenario }\end{array}$} \\
\hline Scenario & Mean & Group \\
\hline S2 Active Shooter & 6.105 & a \\
\hline S3 Building Related & 5.767 & b \\
\hline S5 Robbery & 5.578 & c \\
\hline S1 Snowstorm & 5.550 & c \\
\hline S4 Health Related & 5.416 & d \\
\hline
\end{tabular}

We also compared the means and standard deviations in our study to those in the Han et al. study. Table 8 shows that in general our means and standard deviations are comparable to those in the original study (See Appendix B).

\begin{tabular}{|l|l|l|l|l|l|}
\hline \multicolumn{6}{|c|}{ Table 8. Means and Standard Deviations of Constructs by Scenario } \\
\hline & $\begin{array}{l}\text { S1 } \\
\text { Snowstorm }\end{array}$ & $\begin{array}{l}\text { S2 } \\
\text { Active Shooter }\end{array}$ & $\begin{array}{l}\text { S3 } \\
\text { Building } \\
\text { Related }\end{array}$ & $\begin{array}{l}\text { S4 } \\
\text { Health Related }\end{array}$ & $\begin{array}{l}\text { S5 } \\
\text { Robbery }\end{array}$ \\
\hline & Mean (Std) & Mean (Std) & Mean (Std) & Mean (Std) & Mean (Std) \\
\hline $\begin{array}{l}\text { Subjective } \\
\text { Norm }\end{array}$ & $5.77(1.06)$ & $6.39(0.92)$ & $6.09(0.95)$ & $5.69(1.03)$ & $5.96(0.99)$ \\
\hline $\begin{array}{l}\text { Perceived } \\
\text { Safety Threat }\end{array}$ & $5.21(1.37)$ & $6.37(1.02)$ & $5.79(1.23)$ & $5.59(1.30)$ & $5.60(1.35)$ \\
\hline $\begin{array}{l}\text { Perceived } \\
\text { Financial } \\
\text { Threat }\end{array}$ & $4.03(1.63)$ & $3.75(1.89)$ & $3.53(1.78)$ & $4.00(1.72)$ & $4.28(1.79)$ \\
\hline $\begin{array}{l}\text { Information } \\
\text { Quality Trust }\end{array}$ & $5.41(1.10)$ & $6.11(0.93)$ & $5.77(1.02)$ & $5.55(1.09)$ & $5.58(1.12)$ \\
\hline
\end{tabular}

\section{DISCUSSION}

In general, the results of our study match those of Han, et al. (2015). Table 9 compares the findings of the original study of Han, et al. (2015) and the results of our two analyses (the two-outcome model, and the three-outcome model). It also presents the two dimensions on which Han et al. categorized the nature of incidents: the speed of development (fast or slow) and the area affected (a large area or one limited part of campus). The table shows that our results match well with the Han et al. results, but there are some differences.

\begin{tabular}{|c|c|c|c|c|c|c|c|c|c|c|c|c|c|c|c|}
\hline & \multicolumn{3}{|c|}{$\begin{array}{l}\text { S1 } \\
\text { Snowstorm }\end{array}$} & \multicolumn{3}{|c|}{$\begin{array}{l}\text { S2 } \\
\text { Active Shooter }\end{array}$} & \multicolumn{3}{|c|}{$\begin{array}{l}\text { S3 } \\
\text { Building Related }\end{array}$} & \multicolumn{3}{|c|}{$\begin{array}{l}\text { S4 } \\
\text { Health Related }\end{array}$} & \multicolumn{3}{|c|}{\begin{tabular}{|l|} 
S5 \\
Robbery
\end{tabular}} \\
\hline Speed & \multicolumn{3}{|c|}{ Slow } & \multicolumn{3}{|c|}{ Fast } & \multicolumn{3}{|c|}{ Fast } & \multicolumn{3}{|l|}{ Slow } & \multicolumn{3}{|c|}{ Fast } \\
\hline Area & \multicolumn{3}{|c|}{ Large } & \multicolumn{3}{|c|}{ Limited } & \multicolumn{3}{|c|}{ Limited } & \multicolumn{3}{|c|}{ Large } & \multicolumn{3}{|c|}{ Limited } \\
\hline & Orig & Two & Three & Orig & Two & Three & Orig & Two & Three & Orig & Two & Three & Orig & Two & Three \\
\hline $\begin{array}{l}\text { Past } \\
\text { Experience }\end{array}$ & & & & & & & & & & & & & & & \\
\hline
\end{tabular}




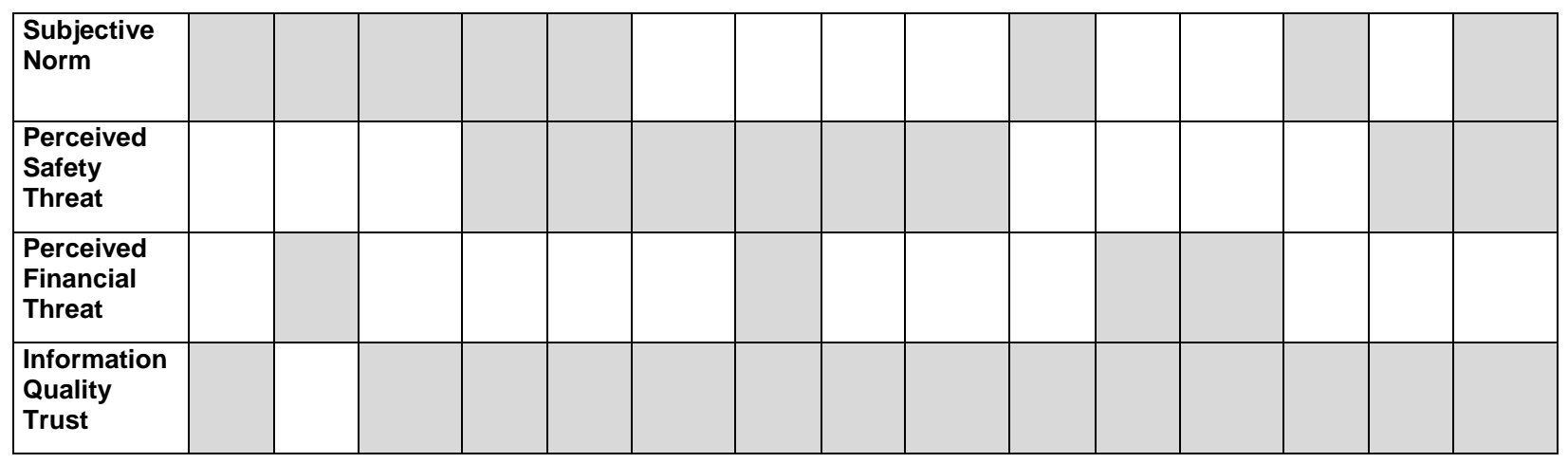

The dominant factor influencing the compliance decision in the original study and both of our analyses was Information Quality Trust. Information quality trust was significant in almost all situations. Thus this replication confirms one of the major takeaways from the original Han, et al. (2015) study: students respond to ENS notifications when they believe they can trust the information they contain. Our post-hoc analyses show that information trust is the dominant factor driving our findings. They also show that students' trust in the ENS is different for different scenarios. So rather than some general overall level of trust in the ENS, students' trust is specific to the type of incident.

Table 8 shows that our students have more trust in the ENS for active shooter and building related incidents, which are fast moving incidents where students have less time to verify the information provided by the ENS before needing to take action, compared to slower moving incidents such as a snowstorm or a health-related incident (Han et al. 2015). The odd one out is the robbery, which is a slow moving incident due to the time it takes for notifications to come in, but our students had less trust in it. Our students reported receiving about two ENS notifications per month during the academic year in which the data were collected, the majority of which were robberies or sexual assaults. In all of these cases, the ENS notification was sent hours after the incident, meaning it had little value as a call to immediate action. In contrast, one ENS notification about a building related incident (a gas leak) occurred in real time while the building was being evacuated, so it provided timely knowledge to act on. We speculate that our students had less trust in ENS about a fast moving robbery event because of their experience with the untimely nature of robbery-related ENS notifications at our campus. Additionally, we note that the ENS notifications on our campus tend to be more detailed than those used in this experiment, as used in Han et al. (2015). While the ENS notifications studied in the original report may have been appropriate for their sample due to similarities between the study ENS and experienced ENS, we speculate that difference in details between the notifications may have caused students to ignore the message. Alerts issued on our campus range from five sentences to multiple paragraphs, while the ENS notifications in the study were one sentence. Research suggests that detailed information is beneficial to users when dealing with emergencies (Sutton et al. 2018). However, given the nature of the five scenarios, providing detailed information in a timely manner may not be always possible (e.g.: detailed investigation by law enforcement agencies for robbery and active shooter scenarios, extent of damage to property for building related incidents and snowstorms).

Additionally, a prior version of the original paper suggested that media richness would positively influence compliance intention (Han et al., 2011). However, at this Midwest university, notifications are often sent out in three different ways - via call, text, and email. When students receive the ENS through these three different routes each time there is an incident, it may actually make the students worry less about the incident, as they perceive that they receive more incidents than actually occur. It is known that habituation to security warnings occurs (Vance et al., 2018), but it not clear how habituation influences attention to warnings when the warnings occur in the ENS context and are related to personal safety.

The implications from this for university administrators are clear. First and foremost, focus on the quality and timeliness of ENS notifications so students learn to trust the information they contain. The other factors in Table 9 may play a role in students' compliance intentions, but the most important lever to influence student compliance is information quality trust.

Table 9 also highlights one difference from the Han, et al. (2015) study. Han, et al., conclude that subjective norms are a "critical" factor (p. 923), especially the norms from parents and university officials, not friends. We found subjective norms to play a role in some situations, but not others, and the pattern was not completely consistent across both the two-outcome and three-outcome models we tested. The relative importance of norms is influenced by culture (Abrams, Ando, and Hinkle, 1998; Fisher, et al., 2009). Some 
cultures put greater emphasis on subjective norms, but there are others which encourage individual perception and discretion (Abrams, et al., 1998; Fisher, et al., 2009). The university studied here has approximately 30 percent more white students than the university that was likely studied in the original paper (Data USA, 2019). This difference in diversity suggests that there may be cultural differences between the two universities. Given the differences in the demographics of the two samples, it is reasonable to assume that cultural differences may have an influence on the importance of subjective norms for these two groups.

Subjective norms were not an overly important factor for our student population, yet they were for Han, et al.'s students. So what's different here? Again, with our university located in the US Midwest, and Han, et al.'s (2015) university in US Northern states, it may be that the difference in diversity influences the concern for subjective norms. The large difference in minority representation may be correlated to a difference in cultural norms for the students. Secondly, past experience with the ENS is an important determinant in creation of subjective norms. Many of our students reported having had a poor past experience with our ENS system, which might have engendered a greater reliance on individual perception and discretion. In our study, since the percentage of students who chose to ignore ENS notifications was higher as compared to that of the original study, we conclude that in emergency situations, our students were less likely to consider subjective norms when making decisions. Also, past experience with ENS not only affects the compliance behavior of students (our subjects) but also affects the compliance behavior of the referents (faculty, staff, etc.). If the referents display less motivation to comply (via informal discussions or as part of general organizational culture), so will the students.

Thus, the second major takeaway from our replication study is that counter to the Han, et al. study, subjective norms are not always "critical." The importance of the subjective norms depends on the students; norms are important for some groups of students, but not for others. One implication for future ENS research is that we need research to better understand students' norms pertaining to ENS, how norms are created, and how norms can be used to make ENS more effective. It may also be relevant to study how the ENS may be better designed to accommodate those of varying cultural and economic background.

The other two ENS levers suggested by Han, et al. that might influence compliance are coercive (safety threats) and remunerative (financial threats). In both the Han, et al. study and in our analyses, Perceived Safety Threat was significant for the Active Shooter and Building fire incidents, both of which present inherent threats to personal safety and are fast moving. In our study, Perceived Safety Threat was also significant for Robbery incidents. On big campuses like ours, there are incidents of robbery reported by our ENS and in the news which may make the students wary of such incidents. However, this particular campus boasts far lower robbery rates than the surrounding municipality, with the city having a lower robbery rate than the US average (Uniform Crime Reporting Statistics, 2019). This (and the lateness of the ENS compared to the event) may also explain the reason why $12.50 \%$ of the participants chose to ignore notifications related to robbery, given the comparatively low frequency of such incidents on this campus.

In both the original study and in our study, perceived financial threat and past experiences with security incidents were noticeably less important than other factors. In the original study, past experience was significant only for building related incidents. In our study, past experience was significant for both snowstorm and building related incidents. Since our study was conducted in the Midwest, the students have some experience with snowstorms, but they especially have experience with the unpredictable nature of our weather. Their previous experience with unpredictable Midwest weather and false alarms for snowstorms is likely what influences their belief in and memory of prior warning messages. Additionally, our campus is quite large with about 125 buildings, so there tends to be a number of building related incidents each year.

In the original study, perceived financial threat was significant only for building related incidents. In our study, perceived financial threat was significant for both snowstorm and health related incidents, but not for building related incidents. We attribute the significance of perceived financial threat for snowstorm incidents to the fact that the liability for the damage to personal property such as cars falls squarely on the individual owners (in our case, students). Also, given the sharp rise in health-care costs in the last 3 years, we expect a natural increase in the significance of perceived financial threat for health related incidents. Thus, past experience and finances may play a role in some situations, but they are not major factors. Additionally, the difference in results for building related incidents is not likely due to the number of students living off campus, as it seems that similar percentages of students live off campus for the North and Midwest universities (US News, 2019). An alternate explanation may be due to perceptions of financial independence between these two groups. Perceptions of financial independence can be influenced by family economic factors (Xiao et al. 2014). If the two groups of students are as different demographically as reports on similar universities 
suggests, then their differences in financial independence may be influencing their perceived financial threat. Financial threat likely is not influenced by the large number of buildings on the campus, as students know they are not responsible for building damage.

In the Han, et al. study, the most common response was to comply immediately; only in the case of healthrelated incidents did a majority of recipients chose to verify first. In our study, the most common response was also to comply immediately, except for health related and snowstorm where the majority of participants chose to first verify the information before complying $(54.79 \%$ for health related and $53.63 \%$ for snowstorm related). The participants in the Han, et al. study noted that the snowstorm and health-related incidents were slower moving, thus suggesting they had more time to watch and respond, so the same may be true of our participants as well.

One other potential explanation for the difference in the results may be past experience for snowstorm incidents in our study. The original study was conducted in the northern states of the US where they may experience greater number of severe snowstorms. In our part of the Midwest, snowstorms tend to be less severe than snowstorms in the northern US, so our recipients may be less likely to comply immediately. Additionally, this part of the Midwest is known for having inaccurate weather reports, with actual weather forecast accuracy $6 \%$ lower across the three most accurate weather providers compared to Buffalo, New York, where the authors of the original paper reside (Forecast Advisor, 2019). The relative inaccuracy of weather forecasts in the area where this campus is located may cause the students to be less trusting of any individual warning. Although, we note that public schools in our town (not the university) were closed twice in the academic year following our study due to snowstorms, so snowstorms are not unknown.

One other interesting difference between the original study and our analyses is the proportion of participants who chose to ignore the notifications. In the original study, less than one percent of all the participants chose to ignore the notifications. In contrast, our study participants said they would ignore the ENS notification about $10 \%$ of the time. This number was significantly higher in three incidents: $15.50 \%$ for health-related, $14.10 \%$ for snowstorm related, and $12.50 \%$ of robbery related. In contrast, participants were less likely to ignore the ENS notification for the two most potentially dangerous, fast-moving events (active shooter: $1.80 \%$; building fire: $3.90 \%$ ).

We included an open-ended question asking participants about other thoughts of our campus ENS, and the responses suggest that there were two main reasons to ignore the notifications: irrelevant information in past ENS notifications and a high frequency of ENS notifications in the past. Participants reported receiving so many ENS notifications that they have become inured to them. This past academic year, our campus averaged two events per month that triggered multiple ENS notifications (e.g., initial notification, one or more situation updates, and an all clear). In many cases, the ENS notifications pertained to a location not near the recipient, so that the notification contained no actionable information. Also, the comments indicated that while ENS notifications informed them about emergency incidents, the notifications (quite rightly) failed to follow-up with regards to the causes or consequences of the incidents, so participants were uncertain as to whether events reported in ENS notifications were actually important. There was a sense of "the boy who cried wolf" among some participants. Therefore, we suggest that future research delve into past experience with ENS notifications (in addition to past experience with emergency events) to better understand how this influences the intention to comply.

The open-ended comments also indicated that some participants believed that government regulations required the university to disclose information through ENS, so the notifications focused more on being correct and equitable over being prompt and in time for action. They cited numerous examples when ENS notifications arrived far too late to enable them to take any meaningful action, suggesting that the ENS notifications were not useful, but were instead intended to protect the university. Therefore, we suggest that future research consider the timeliness of past ENS notifications to better understand how ENS notifications influence the intention to comply.

The results of this replication suggest that there is substantial opportunity to better understand how culture may affect response to ENS. These results suggest that culture may have a large impact on response to ENS warnings, leading to the conclusion that the ENS needs to be designed toward the population of interest. Additionally, there is opportunity to bridge the research on ENS with recent research on security warnings to better present the ENS warnings to be maximally effective for the target population, whether students at a university or residents in an area with possible natural disasters. 


\section{Conclusion}

Han, et al. (2015) concluded that compliance with campus ENS notifications was driven primarily by two factors: trust in the quality of the ENS information, and subjective norms. Our replication also concludes that information quality trust is important, but found less support for subjective norms. Our participants were drawn from a different part of the US, so we speculate that the importance of subjective norms may depend upon culture, which may differ from region to region, and also upon a general organizational disposition towards ENS systems.

As in the Han, et al. study, past experience with security incidents, personal safety and financial threats played a more modest role, influencing compliance in some situations - those that are fast-moving and highly dangerous (e.g., building fire, active shooter) - but lacking a strong effect in all situations.

Our study suggests one additional factor that should be included in future research - the nature of ENS notifications that students have experienced, and especially their timeliness. Also, with the sharp increase in the variety of communication systems preferred by the students and other members of educational institutions, it is important to study the most efficient mode of communication especially during emergency situations. If authorities fail to have a real-time presence in modern and preferred modes of communication, there is a higher risk of dissemination of illegitimate and panic-causing information. With advancement in technology and social media, students now have access to real-time resources for obtaining information. In the future, increased access to information may lead to increased propensity to verify first before complying, which may become a universal and inevitable new normal. As a corollary, the ability of social media to capture "unreported" incidents poses further challenges for campus authorities to improve information quality trust in ENS notifications. Lastly, for big campuses and campuses spread out within the cities, the consideration of proximity of the recipients to the location of emergency incidents hold higher importance. The study of the relevance of using Geographical Information Systems in tandem with ENSs could help optimize the reach of the ENSs to the correct recipients in a timelier manner.

Information Systems succeed when they are created as a result of high demand for consumption of information. ENS systems may be different because they are created not as a result of demand for consumption of information, but as a result of a regulatory requirement. Regulatory requirements typically mandate creation of descriptive reports and not necessarily mandate providing actionable insights. It is our hope that this unique case of ENS systems instigates further research on success of regulatory requirements of information systems.

Lastly, it will be interesting to study if the compliance behavior of students (subjects) changes if they are made party during the decision making process for information dissemination. For starters, students can be given options to choose their preferred medium of communication for specific type of emergency incidents. For example, a student not living on campus may choose to not receive phone calls for incidents that happen on campus. In which case, if a student does receive a phone call from the ENS for other emergency situations, their level of compliance may increase. 


\section{References}

Abrams, D., Ando, K., and Hinkle, S. (1998). Psychological attachment to the group: Cross-cultural differences in organizational identification and subjective norms as predictors of workers' turnover intentions. Personality and Social Psychology Bulletin, 24(10), 1027-1039.

Data USA (2019). Data USA. https://datausa.io/. Accessed on Feb 26, 2019.

Etzioni, A. (1961). Compliance, goals, and effectiveness. in A Comparative Analysis of Complex Organizations, A. Etzioni (ed.), New York: Free Press, 71-88.

Fisher, R., Ferreira, M.C., Assmar, E., Redford, P., Harb, C., Glazer, S., Chaeng, B.S., Jiang, D.Y., Wong, C.C., Kumar, N., Kartner, J., Hofer, J., and Achoui, M. (2009). Individualism-collectivism as descriptive norms: Development of a subjective norm approach to culture measurement. Journal of Cross-Cultural Psychology, 40(2), 187-213.

Forecast Advisor (2019). Weather forecast accuracy details. https://www.forecastadvisor.com/. Accessed on Feb 26, 2019.

Han, W., Ada, S., Sharman, R., Rao, H. R., \& Brennan, J. (2011). Critical success factors to improve compliance with campus emergency notifications. Paper presented at the Americas Conference on Information Systems.

Han, W., Ada, S., Sharman, R., and Rao, H.R. (2015). Campus emergency notification systems: An examination of factors affecting compliance with alerts. MIS Quarterly. 39(4), 909-929.

Kutner, M. H., Nachtsheim, C. J., and Neter, J. (2004). Applied Linear Regression Models. (4th ed.) McGraw-Hill Irwin.

Mitroff, I., Diamond, M., and Alpaslan, M. (2006). How prepared are America's colleges and universities for major crises? Change: The Magazine of Higher Learning. 38(1), 61-67.

Parsons, T. 1957. Illness and the role of the physician: A sociological perspective. American Journal of Orthopsychiatry. 21(4), 452-460.

Sutton, J., Vos, S., Wood, M., and Turner, M. (2018). Designing effective tsunami messages: Examining the role of short messages and fear in warning response. Weather, Climate, and Society. 10, 75-87.

Uniform Crime Reporting Statistics. (2019). UCR. https://www.ucrdatatool.gov/. Accessed Feb 26, 2019.

Vance, A., Jenkins, J. L., Anderson, B. B., Bjornn, D. K., \& Kirwan, C. B. (2018). Tuning out security warnings: A longitudinal examination of habituation through $\mathrm{fMRI}$, eye tracking, and field experiments. MIS Quarterly, 42(2), 355-380.

Xiao, J. J., Chatterjee, S., and Kim, J. (2014). Factors associated with financial independence of young adults. International Journal of Consumer Studies. 38, 394-403. 


\section{Appendix A: Constructs and Items}

\begin{tabular}{|l|l|}
\hline Construct & Incident Questions \\
\hline $\begin{array}{l}\text { Subjective Norm (Motivation to Comply with the } \\
\text { Referent } \times \text { Normative Belief) }\end{array}$ & \\
\hline $\begin{array}{l}\text { Motivation to Comply with the } \\
\text { Referent }\end{array}$ & $\begin{array}{l}\text { Given the scenario above, I would care what _ } \\
\text { should do. }\end{array}$ \\
\hline $\begin{array}{l}\text { Motivation to Comply with the } \\
\text { Referent 1 }\end{array}$ & my friends \\
\hline $\begin{array}{l}\text { Motivation to Comply with the } \\
\text { Referent 2 }\end{array}$ & my parents \\
\hline $\begin{array}{l}\text { Motivation to Comply with the } \\
\text { Referent 3 }\end{array}$ & university officials \\
\hline $\begin{array}{l}\text { Motivation to Comply with the } \\
\text { Referent 4 }\end{array}$ & my professors \\
\hline $\begin{array}{l}\text { Motivation to Comply with the } \\
\text { Referent 5 }\end{array}$ & other people who are important to me \\
\hline Normative Belief & $\begin{array}{l}\text { Given the scenario above } \\
\text { comply immediately with the campus alert. }\end{array}$ \\
\hline Normative Belief 1 & my friends \\
\hline Normative Belief 2 & my parents \\
\hline Normative Belief 3 & university officials \\
\hline Normative Belief 4 & my professors \\
\hline Normative Belief 5 & other people who are important to me \\
\hline Perceived Safety Threat & The scenario above could ... \\
\hline Perceived Self Safety Threat 1 & have a severe impact on my safety. \\
\hline Perceived Self Safety Threat 2 & get me hurt or injured. \\
\hline Perceived Financial Threat & It is likely to ... \\
\hline Perceived Financial Threat 1 & have a serious impact on my finances. \\
\hline Perceived Financial Threat 2 & cause me monetary loss. \\
\hline
\end{tabular}




\begin{tabular}{|l|l|}
\hline Construct & Question \\
\hline Information Quality Trust & $\begin{array}{l}\text { Given the above scenario, how much would you agree with the following } \\
\text { statements regarding campus alerts? }\end{array}$ \\
\hline Information Relevance Trust 1 & The alert would be relevant to me. \\
\hline Information Relevance Trust 2 & The alert would be sent only when necessary. \\
\hline Information Relevance Trust 3 & The alert would be important for me to make decisions about the situation. \\
\hline Information Actionability Trust 1 & I can act on the information that I received in the alert. \\
\hline Information Actionability Trust 2 & If I follow the instructions in the alert, I will be protected. \\
\hline Information Actionability Trust 3 & The directions in the alert will help me plan my next step. \\
\hline Information Criticality Trust 1 & The timing of the alert would be appropriate \\
\hline Information Criticality Trust 2 & The alert I received conveys the urgency for taking action. \\
\hline Information Criticality Trust 3 & The alert I received conveys the severity of the incident. \\
\hline $\begin{array}{l}\text { ENS-Message Compliance } \\
\text { Intention }\end{array}$ & $\begin{array}{l}\text { Given the above scenario with the emergency notification message asking you } \\
\text { to take an action, what are you likely to do first? } \\
\text { Comply immediately } \\
\text { Verify first and then comply } \\
\text { Ignore }\end{array}$ \\
\hline
\end{tabular}

Note: Subjective Norm, Perceived Safety Threat, Perceived Financial Threat, and Information Quality Trust are measured on a seven-point Likert scale. The scales are fully anchored (strongly disagree, disagree, somewhat disagree, neither agree nor disagree, somewhat agree, agree, strongly agree). 


\begin{tabular}{|l|l|}
\hline Construct & Demographic Questions \\
\hline Gender & $\begin{array}{l}\text { What is you gender? } \\
\text { Male Female }\end{array}$ \\
\hline Age & What is your age? \\
\hline Ethnicity & $\begin{array}{l}\text { Would you describe yourself as: } \\
\text { White/Caucasian (not Hispanic) } \\
\text { Hispanic } \\
\text { Black/African American } \\
\text { Asian } \\
\text { Native American } \\
\text { Pacific Islander } \\
\text { Other }\end{array}$ \\
\hline Citizenship & $\begin{array}{l}\text { Are you a } \\
\text { US Citizen Non-US Citizen }\end{array}$ \\
\hline Student type & $\begin{array}{l}\text { Are you an } \\
\text { Undergraduate Student Graduate Student }\end{array}$ \\
\hline Past Experience 1 & $\begin{array}{l}\text { How many campus emergency alerts have you received since you } \\
\text { became a student at IU? } \\
0\end{array}$ \\
\hline Past Experience 2 & $\begin{array}{l}\text { Have you ever experienced any type of incident on campus? } \\
\text { Yes No }\end{array}$ \\
\hline Past Experience 3 & Do you have any comments on the IU campus emergency alert system? \\
\hline
\end{tabular}




\section{Appendix B}

To obtain overall means for the constructs in Han et al. 2016, we averaged the means and standard deviations for the items within each construct. See the comparison between those means and the means from this study in the table below.

\begin{tabular}{|c|c|c|c|c|c|c|c|c|c|c|}
\hline & $\begin{array}{l}\text { S1 } \\
\text { Snow- } \\
\text { storm }\end{array}$ & $\begin{array}{l}\text { Han et al. } \\
\text { S1 }\end{array}$ & \begin{tabular}{|l} 
S2 \\
Active \\
Shooter
\end{tabular} & \begin{tabular}{|l|} 
Han et al. \\
S2
\end{tabular} & \begin{tabular}{|l|} 
S3 \\
Building \\
Related
\end{tabular} & $\begin{array}{|ll|}\text { Han } & \text { et } \\
\text { al. } & \\
\text { S3 } & \\
\end{array}$ & \begin{tabular}{|l|} 
S4 \\
Health \\
Related
\end{tabular} & $\begin{array}{l}\text { Han et al. } \\
\text { S4 }\end{array}$ & $\begin{array}{l}\text { S5 } \\
\text { Robbery }\end{array}$ & $\begin{array}{ll}\text { Han } & \text { et } \\
\text { al. } & \\
\text { S5 } & \end{array}$ \\
\hline & $\begin{array}{l}\text { Mean } \\
\text { (Std) }\end{array}$ & $\begin{array}{l}\text { Mean } \\
\text { (Std) }\end{array}$ & $\begin{array}{l}\text { Mean } \\
\text { (Std) }\end{array}$ & $\begin{array}{l}\text { Mean } \\
\text { (Std) }\end{array}$ & Mean (Std) & $\begin{array}{l}\text { Mean } \\
\text { (Std) }\end{array}$ & $\begin{array}{l}\text { Mean } \\
\text { (Std) }\end{array}$ & $\begin{array}{l}\text { Mean } \\
\text { (Std) }\end{array}$ & Mean (Std) & $\begin{array}{l}\text { Mean } \\
\text { (Std) }\end{array}$ \\
\hline $\begin{array}{l}\text { Subjective } \\
\text { Norm }\end{array}$ & $\begin{array}{l}5.77 \\
(1.06) \\
\end{array}$ & $\begin{array}{l}5.82 \\
(1.18) \\
\end{array}$ & $\begin{array}{l}6.39 \\
(0.92) \\
\end{array}$ & $\begin{array}{l}5.85 \\
(1.12) \\
\end{array}$ & $6.09(0.95)$ & $\begin{array}{l}5.68 \\
(1.26) \\
\end{array}$ & $\begin{array}{l}5.69 \\
(1.03) \\
\end{array}$ & $\begin{array}{l}5.92 \\
(1.19) \\
\end{array}$ & $5.96(0.99)$ & $\begin{array}{l}5.76 \\
(1.26) \\
\end{array}$ \\
\hline $\begin{array}{l}\text { Perceived } \\
\text { Safety } \\
\text { Threat }\end{array}$ & $\begin{array}{l}5.21 \\
(1.37)\end{array}$ & $5.84(1.3)$ & $\begin{array}{l}6.37 \\
(1.02)\end{array}$ & \begin{tabular}{|l|}
6.46 \\
$(0.95)$
\end{tabular} & $5.79(1.23)$ & $\begin{array}{l}5.87 \\
(1.15)\end{array}$ & $\begin{array}{l}5.59 \\
(1.30)\end{array}$ & $\begin{array}{l}5.39 \\
(1.59)\end{array}$ & $5.60(1.35)$ & $\begin{array}{l}5.92 \\
(1.19)\end{array}$ \\
\hline $\begin{array}{l}\text { Perceived } \\
\text { Financial } \\
\text { Threat }\end{array}$ & $\begin{array}{l}4.03 \\
(1.63)\end{array}$ & $\begin{array}{l}4.36 \\
(1.68)\end{array}$ & $\begin{array}{l}3.75 \\
(1.89)\end{array}$ & $\begin{array}{l}3.90 \\
(1.92)\end{array}$ & $3.53(1.78)$ & $\begin{array}{l}4.58 \\
(1.77)\end{array}$ & $\begin{array}{l}4.00 \\
(1.72)\end{array}$ & $\begin{array}{l}4.56 \\
(1.74)\end{array}$ & $4.28(1.79)$ & $\begin{array}{l}4.81 \\
(1.78)\end{array}$ \\
\hline $\begin{array}{l}\text { Information } \\
\text { Quality } \\
\text { Trust }\end{array}$ & \begin{tabular}{|l|}
5.41 \\
$(1.10)$
\end{tabular} & $\begin{array}{l}6.05 \\
(1.12)\end{array}$ & $\begin{array}{l}6.11 \\
(0.93)\end{array}$ & \begin{tabular}{|l|}
6.23 \\
$(1.05)$
\end{tabular} & $5.77(1.02)$ & $\begin{array}{l}6.14 \\
(1.00)\end{array}$ & \begin{tabular}{|l|}
5.55 \\
$(1.09)$
\end{tabular} & $\begin{array}{l}6.06 \\
(0.94)\end{array}$ & $5.58(1.12)$ & $\begin{array}{l}6.03 \\
(1.15)\end{array}$ \\
\hline
\end{tabular}




\section{About the Authors}

Rishikesh Chetan Muchhala is a Chicago-based management consultant with PricewaterhouseCoopers LLP in the Health Industries business. Rishi holds a Master of Science in information systems from the Kelley School of Business at Indiana University Bloomington. While he dons a practitioner's hat in his day job, his research interests include societal impact of technology, adoption of information systems, misuse, underuse, and wastage of technology.

Patricia L. Moravec. (ORCID: 0000-0001-5896-5444) is an assistant professor of Information Management at the McCombs School of Business, University of Texas at Austin. Patricia earned her Ph.D. in Information Systems from the Kelley School of Business at Indiana University, as well as a Masters in Information Systems. Her undergraduate degree was in physics and astronomy/astrophysics from Indiana University. Her research interests include information quality, fake news and the cognition behind believing fake information on social media, and disaster response on social media. She previously served as the managing editor for MIS Quarterly Executive. She has also published in Management Information Systems Quarterly and Journal of Management Information Systems. 